\title{
Breast implant-associated anaplastic large cell lymphoma
}

Natasha Patrick, Debra Meerkotter

\section{CASE}

A female patient aged 75 years had undergone bilateral mastectomy and reconstruction with silicone implants following breast cancer seven years ago. She developed a sudden significant swelling of the right reconstructed breast. She presented for ultrasonography of the reconstructed breast. The ultrasound revealed a periprosthetic fluid collection (Figure 1).

Clinically, there was marked asymmetry of the breasts: the right breast was swollen, and the skin was tightly stretched over the implant. Ultrasound-guided aspiration of the fluid was performed, draining $230 \mathrm{~mL}$ of straw-coloured fluid. The fluid was sent for culture, cytology and flow cytometry. Flow cytometry demonstrated a pleomorphic large cell population with cell marker analysis favouring T-cell lymphoproliferative disorder in keeping with breast implant-associated anaplastic large cell lymphoma (BIA-ALCL).

QUESTION 1

What is BIA-ALCL?

QUESTION 2

What are the signs and symptoms?

QUESTION 3

What are appropriate investigations?

QUESTION 4

What are typical findings on imaging?
QUESTION 5

What are the pathological findings consistent with diagnosis?

\section{QUESTION 6}

What is the aetiology of BIA-ALCL?

\section{ANSWER 1}

BIA-ALCL is a rare form of T-cell lymphoma associated with breast implants. This entity commonly presents with an effusion developing between the breast implant and the fibrous capsule that surrounds it. It can present either as an effusion alone or with a mass lesion. ${ }^{1}$ It is characterised by abnormal growth of lymphocytes with a strong expression of CD30 that arise within the peri-implant capsule, and predominantly presents as an effusion with sudden breast swelling. ${ }^{2}$

\section{ANSWER 2}

A patient may notice breast enlargement or asymmetry, warmth and pain at least one-year post-surgery. ${ }^{3}$ On clinical examination, there may be breast enlargement due to the seroma and/or a palpable mass around the implant or in the axilla. ${ }^{4}$

\section{ANSWER 3}

Recent breast changes in a person with breast implants should receive a full clinical breast examination and ultrasonography. ${ }^{5}$ Ultrasonography can be used to guide fluid aspiration or core biopsy of any solid masses. In BIA-ALCL, CD30 staining from the fluid aspirate is key to diagnosis. Magnetic resonance imaging (MRI) of the breast (Figure 2) with contrast and positron emission tomography (PET) scanning is used for staging of diagnosed cases of BIA-ALCL (with Medicare Benefits Schedule rebate). ${ }^{4}$

\section{ANSWER 4}

Ultrasonography can confirm a peri-implant fluid collection. The volume of fluid can range 50-1000 mL. ${ }^{6}$ Associated masses and lymphadenopathy can also be assessed. Minimal peri-implant fluid is a normal finding and does not require investigation unless clinically indicated. ${ }^{6}$

MRI is useful for assessing for a peri-implant fluid collection (Figures 2 and 3). Intravenous gadolinium contrast will allow for detection of associated enhancing masses. Silicone implant integrity can also be evaluated with silicone-weighted imaging (Figure 4).

PET is useful for staging of disease. The effusion demonstrated moderate fluorodeoxyglucose (FDG) uptake. Any associated mass, pathological lymph nodes or metastatic disease will show FDG uptake. ${ }^{6}$

\section{ANSWER 5}

Cytology demonstrates large, pleomorphic cells. Characteristic 'horseshoe-shaped' nuclei may be seen. On immunohistochemical staining there is CD30 positivity. ${ }^{6}$ BIA-ALCL is typically anaplastic lymphoma kinase (ALK) negative. ${ }^{6}$

\section{ANSWER 6}

The disease appears more commonly in textured implants. There appears to be a chronic inflammatory response that produces chronic antigen stimulation and progresses to malignant transformation 
of activated T-cells. ${ }^{1}$ The chronic inflammation may in part be related to subclinical infection. ${ }^{6}$

There appears to be no difference between saline and silicone implants. The risk is not altered if the surgery was performed for reconstruction post-breast cancer surgery or for primary reconstruction. Implant rupture does not increase risk. ${ }^{6}$

\section{CASE CONTINUED}

The patient was referred for surgical excision of both implants and surrounding fibrous capsule (capsulectomy). She has received surveillance computerised tomography scans and remains clinically free of recurrent disease.

Patients who present with late onset seroma, such as this woman, have a

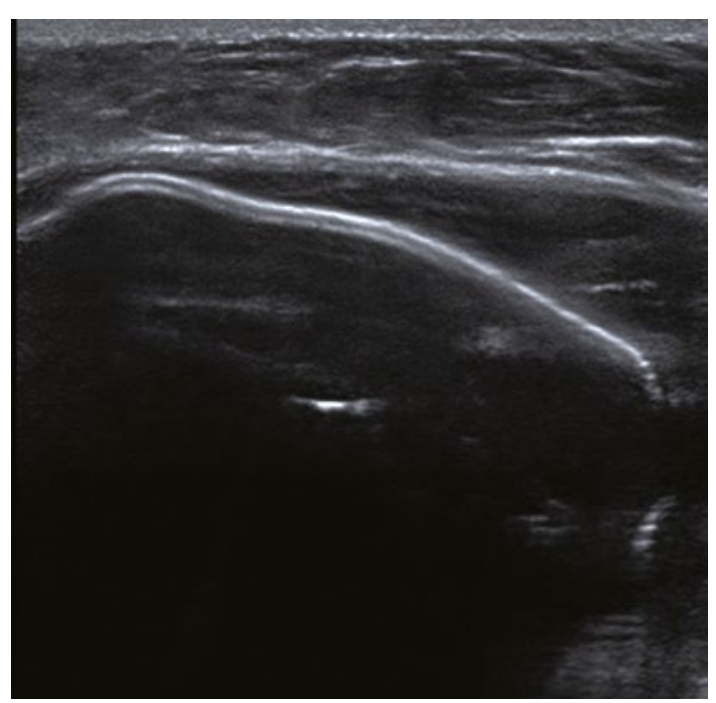

Figure 1. Ultrasonography of the right reconstructed breast. There is a silicone implant, surrounding which is a fluid collection.

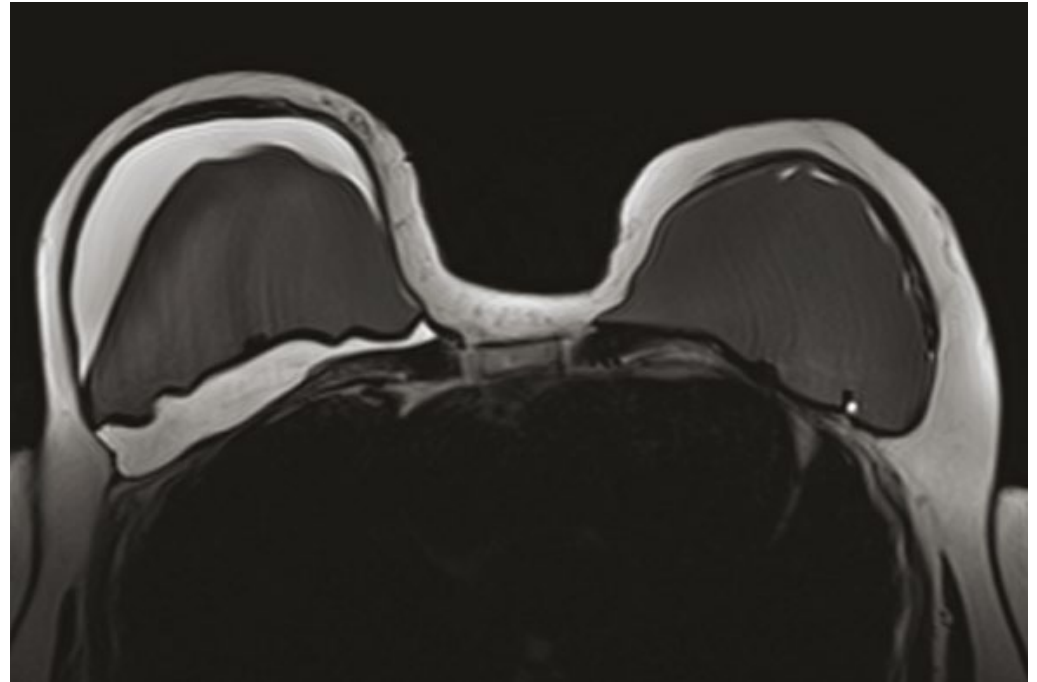

Figure 2. Axial breast magnetic resonance imaging. This study is water weighted, producing a hyperintense appearance of the fluid around the right implant. The study has suppressed the signal from silicone, producing a hypointense appearance of the implants.

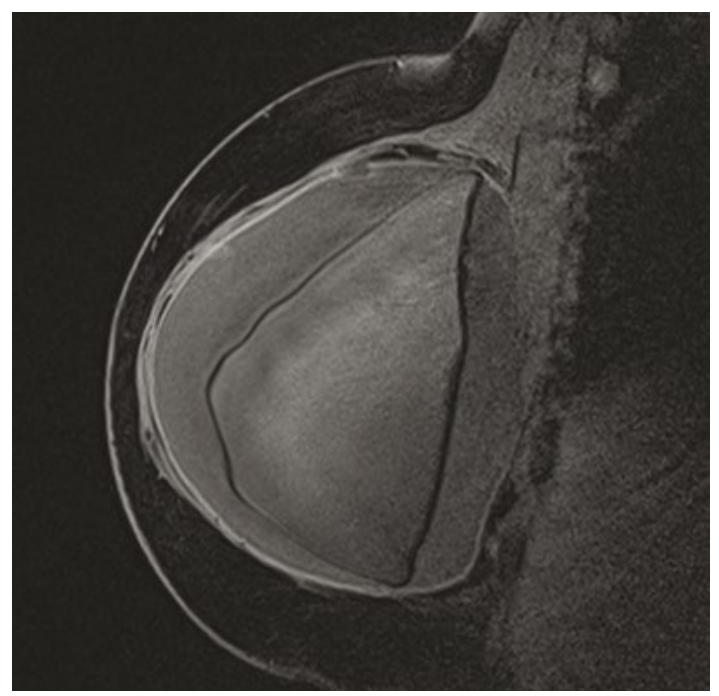

Figure 3. Sagittal magnetic resonance imaging of the right reconstructed breast, which confirms the peri-implant fluid collection. It also demonstrates that this implant has been placed in a retro-pectoral position.

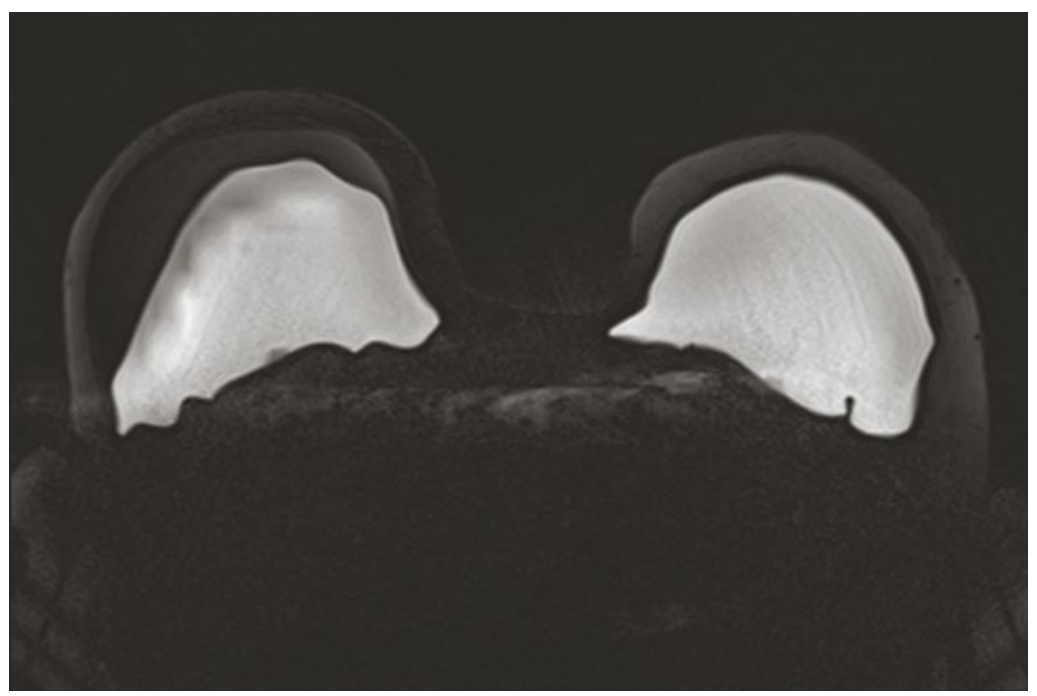

Figure 4. Axial silicone-weighted study. This demonstrates intact silicone implants. 
good prognosis with surgical removal and total capsulectomy. ${ }^{6}$ In patients who present with a mass or extensive disease, the prognosis is worse and adjuvant chemotherapy, radiotherapy and surgery may be required. ${ }^{6,7}$

\section{Key points}

- Implant-associated effusion more than a year after surgery must raise suspicion for BIA-ALCL.

- Ultrasonography is a recommended imaging modality to assess for effusion, masses and lymphadenopathy. Ultrasound-guided aspiration of fluid for culture, cytology and flow cytometry is critical for diagnosis.

- Improved awareness of this entity and recommended investigations may aid early detection and improve prognosis.
- This disease entity is thought to be underreported. The number of cases continues to grow.

- Patients should be made aware of this uncommon disease prior to implant surgery.

\section{Authors}

Natasha Patrick, University of Tasmania, Tas Debra Meerkotter MBBCh, FCRad (D) SA, FRANZCR, Radiologist, I-MED Radiology, Tas

Competing interests: None.

Funding: None.

Provenance and peer review: Not commissioned, externally peer reviewed.

\section{Correspondence to:}

deband67@gmail.com

\section{References}

1. Thompson PA, Prince HM. Breast implantassociated anaplastic large cell lymphoma: A systematic review of the literature and mini-meta analysis. Curr Hematol Malig Rep 2013;8(3):196-210. doi: 10.1007/s11899-0130164-3.
2. Rupani A, Frame JD, Kamel D. Lymphomas associated with breast implants: A review of the literature. Aesthet Surg J 2015;35(5):533-44. doi: 10.1093/asj/sjv016.

3. Bizjak M, Selmi C, Praprotnik S, et al. Silicone implants and lymphoma: The role of inflammation. J Autoimmun 2015;65:64-73. doi: 10.1016/j.jaut.2015.08.009.

4. American Society of Plastic Surgeons. Breast implant associated anaplastic large cell lymphoma (BIA-ALCL). Arlington Heights, IL: ASPS, 2020. Available at www.plasticsurgery. org/patient-safety/breast-implant-safety/biaalcl-summary [Accessed 22 July 2020].

5. Lourenco AP, Moy L, Baron P, et al. ACR Appropriateness Criteria ${ }^{\circledR}$ Breast Implant Evaluation. J Am Coll Radiol 2018;15(5S):S13-S52. doi: 10.1016/j.jacr.2018.03.009.

6. Gunawardana RT, Dessauvagie BF, Taylor DB. Breast implant-associated anaplastic large cell lymphoma, an under-recognised entity. $J$ Med Imaging Radiat Oncol 2019;63(5):630-38. doi: 10.1148/radiol.2018160440.

7. Aladily TN, Mederios LJ, Amin MB, et al. Anaplastic large cell lymphoma associated with breast implants: A report of 13 cases. Am J Surg Pathol 2012;36(7):1000-08. doi: 10.1097/ PAS.0b013e31825749b1. 